\title{
Dynamic Chemical Communication between Plants and Bacteria through Airborne Signals: Induced Resistance by Bacterial Volatiles
}

\author{
Mohamed A. Farag • Huiming Zhang • Choong-Min Ryu
}

Received: 17 April 2013 / Revised: 18 June 2013 / Accepted: 27 June 2013 / Published online: 24 July 2013

(C) The Author(s) 2013. This article is published with open access at Springerlink.com

\begin{abstract}
Certain plant growth-promoting rhizobacteria (PGPR) elicit induced systemic resistance (ISR) and plant growth promotion in the absence of physical contact with plants via volatile organic compound (VOC) emissions. In this article, we review the recent progess made by research into the interactions between PGPR VOCs and plants, focusing on VOC emission by PGPR strains in plants. Particular attention is given to the mechanisms by which these bacterial VOCs elicit ISR. We provide an overview of recent progress in the elucidation of PGPR VOC interactions from studies utilizing transcriptome, metabolome, and proteome analyses. By monitoring defense gene expression patterns, performing 2dimensional electrophoresis, and studying defense signaling null mutants, salicylic acid and ethylene have been found to be key players in plant signaling pathways involved in the ISR response. Bacterial VOCs also confer induced systemic tolerance to abiotic stresses, such as drought and heavy metals. A review of current analytical approaches for PGPR volatile profiling is also provided with needed future developments emphasized. To assess potential utilization of PGPR VOCs for crop plants, volatile suspensions have been applied to pepper and cucumber roots and found to be effective
\end{abstract}

M. A. Farag $(\varangle)$

Pharmacognosy Department, Faculty of Pharmacy, Cairo

University, Cairo 11562, Egypt

e-mail:mfarag73@yahoo.com

\section{H. Zhang}

Department of Horticulture and Landscape Architecture, Purdue University, West Lafayette, IN 47907, USA

C.-M. Ryu ( $\square)$

Molecular Phytobacteriology Laboratory, Superbacteria Research Center, KRIBB, Daejeon 305-806, South Korea

e-mail: cmryu@kribb.re.kr

C.-M. Ryu

Biosystems and Bioengineering Program, University of Science and Technology, Daejeon 305-350, South Korea at protecting plants against plant pathogens and insect pests in the field. Taken together, these studies provide further insight into the biological and ecological potential of PGPR VOCs for enhancing plant self-immunity and/or adaptation to biotic and abiotic stresses in modern agriculture.

Keywords PGPR · ISR · IST · Volatile organic compounds · Headspace
Abbreviations
PGPR Plant growth-promoting rhizobacteria
ISR Induced systemic resistance
IST Induced systemic tolerance
VOC Volatile organic compound

\section{Introduction}

Plants and microorganisms abound with natural chemicals, many of which are volatiles. These molecules are chemically diverse, representing fatty acid derivatives, terpenes, indoles, and molecules from other chemical families (Paré and Tumlinson 1999). Among these compounds, ethylene (ET, $\mathrm{C}_{2} \mathrm{H}_{4}$ ), a potent activator of plant defense responses, was the first gaseous hormone discovered in nature (Bleeker and Kende 2000). New, fundamental insights also have emerged from examining other plant volatiles that can act as signaling entities in plants and microorganisms. The airborne natural product derivative methyl jasmonate (MeJA), which is released from plant tissues upon exposure to insect or mechanical damage, triggers proteinase inhibitor and polyphenol oxidase induction in undamaged tomatoes or in neighboring plants (Lycopersicon esculentum; Fidantsef et al. 1999) and induces phytoalexin accumulation in bean and barley (Hordeum vulgare; Croft et al. 1993; Weidhase et al. 1987). Other examples of plant volatile chemicals that 
trigger plant defense responses include the induction of defense-related gene expression by methyl salicylate (MeSA) in tobacco (Nicotiana tabacum; Shulaev et al. 1997). When certain C6 components produced from a branch of the lipoxygenase (LOX) pathway (Hatanaka et al. 1987) are released, these compounds can reduce herbivore infestation (Hildebrand et al. 1993), seed germination frequency (Gardener et al. 1990), and phytoalexin induction in nearby plants (Zeringue 1992). Molecular data have demonstrated that aerial treatment of Arabidopsis and lima bean with the synthetic C6-volatile $(E)$-2-hexenal induces the transcription of defense-related genes (Arimura et al. 2000; Bate and Rothstein 1998). However, while bacterial volatile determinants are known to also trigger secondary responses in planta, little is known about their role in regulating plant growth and/or defense responses. The observation that volatile emissions from bacteria can interact with plants has only recently been reported.

Plant Growth is Promoted by Bacterial "Odors" In 2003, Ryu et al. discovered that a blend of airborne chemicals released from specific bacterial strains of plant growth-promoting rhizobacteria (PGPR) can promote the growth of Arabidopsis thaliana seedlings. PGPR include a wide range of rootcolonizing bacteria with the capacity to enhance plant growth by increasing seed emergence, plant weight, and crop yields (Kloepper 1992). Seed or seedling treatment with PGPR has been used to increase the growth of several crops (Glick 1995) and to suppress the growth of plant pathogens and deleterious rhizosphere microorganisms. In addition to positively affecting plant growth, the application of certain PGPR strains to seeds or seedlings leads to a state of induced systemic resistance (ISR) that is a type of induced resistance elicited by PGPR. In contrast, pathogen- or chemical trigger-mediated induced resistance is often referred to as systemic acquired resistance (SAR) (Kloepper et al. 1999; Pieterse et al. 2002; 2009). ISR has been triggered in several crops against fungal, bacterial, and viral pathogens under both greenhouse and/or field conditions (Kloepper et al. 1999, 2004; Liu et al. 1995; Murphy et al. 2003; Ryu et al. 2004a, b, c; Zehnder et al. 1999). Recently, several genera of PGPR strains also were found to promote plant growth and elicit ISR via bacterial volatile emissions under in vitro conditions (Ryu et al. 2003, 2004a, b, c). The current review focuses primarily on the role of volatiles produced by the Gram positive PGPR strains: Bacillus subtilis GB03, Bacillus amyloliquefaciens IN937a, and Paenibacillus polymyxa E681 and one Gram negative strain Pseudomonas chlororaphis $\mathrm{O} 6$ in inducing plant immune responses. Few previous reports have focused on Gram positive bacterial VOCelicited plant immune responses. Rather, more attention has been on describing Gram negative bacterial-mediated plant growth alteration (Effmert et al. 2012; Han et al. 2006; Ryu et al. 2004a; Wenke et al. 2010).
Initially, in the process of developing an assay system to assess the growth promotion capacity of rhizobacteria in vitro (Ryu et al. 2003), we found that bacterial volatiles probably are involved in plant growth promotion. An assessment of growth promotion induced by bacterial volatiles in Arabidopsis revealed that inoculation with the GB03 or IN937a strain significantly promoted the growth of Arabidopsis compared with the water control or treatment with DH $5 \alpha$. We assayed the level of plant growth promotion by PGPR VOCs under laboratory conditions by physically separating seedlings from PGPR on divided Petri dishes (referred to as I-plates, Fig. 1); this treatment allowed only airborne signals to be transmitted between the bacterial cultures and the seedlings (Ryu et al. 2003, 2004a). The two most abundant compounds released from cultures of strains GB03 and IN937a, albeit not from cultures of the other strains, were identified as 2,3-butanediol and its precursor 3hydroxy-2-butanone (syn. acetoin; Fig. 2)(Ryu, et al. 2003, 2004a). The qualitative and quantitative compositions of volatile blends emitted by the growth-promoting strains differ significantly from those of the null growth-promoting bacterium DH5 $\alpha$ (Ryu et al. 2003). Exogenous application of commercial acetoin and 2,3-butanediol result in the dosedependent stimulation of plant growth, which simulates the effects of the volatile blend produced by the two Bacillus sp.. Volatiles released from mutant strains of $B$. subtilis that are genetically blocked in the production of 2,3-butanediol have no effect on plants, which confirms the role of these compounds as plant growth-promoting volatile determinants (Ryu et al. 2003). Considering that these active compounds i.e., 2,3-butanediol and acetoin were elucidated only from an in vitro setup, further experiments should be conducted in

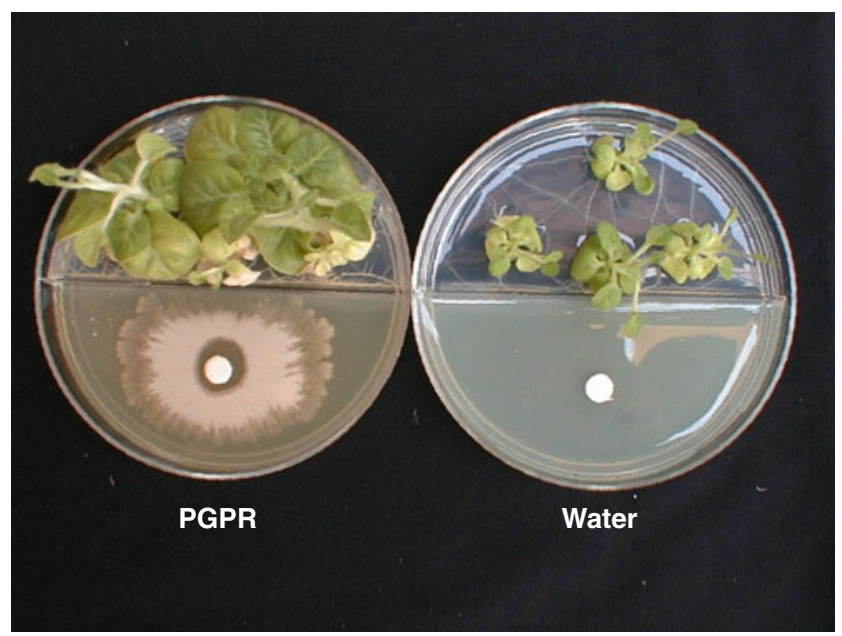

Fig. 1 I-plate system used for assessing plant growth promotion in response to plant growth-promoting rhizobacteria (PGPR) volatiles exposure. This setup allows only volatile compounds to be exchanged, while preventing any diffusion of non-volatile metabolites through the medium 

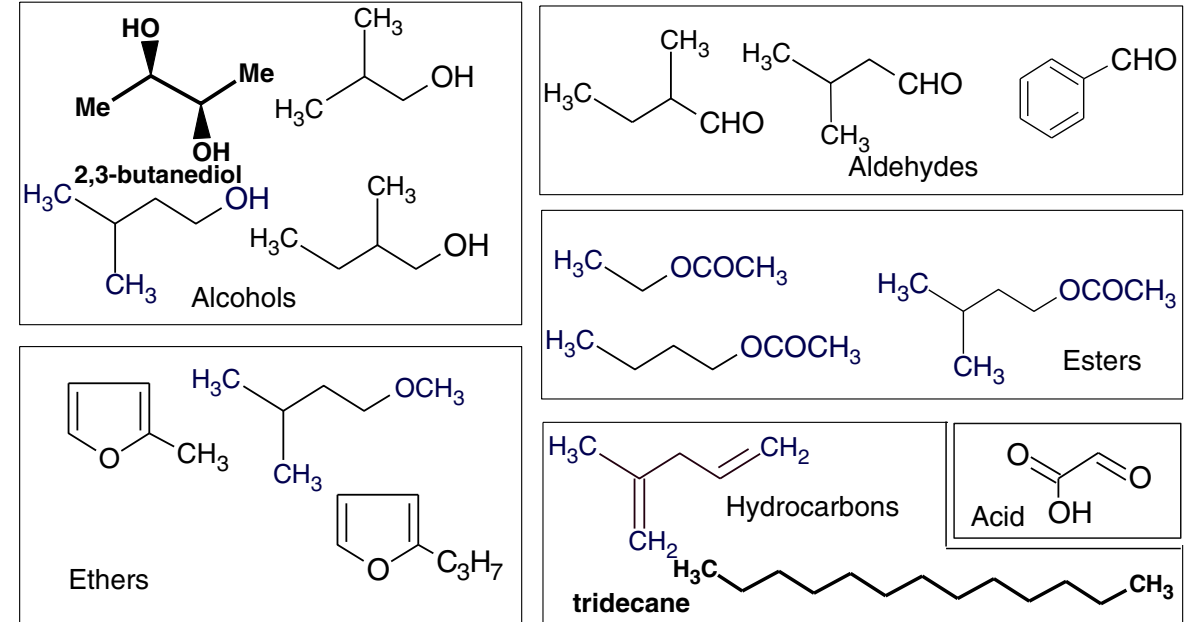

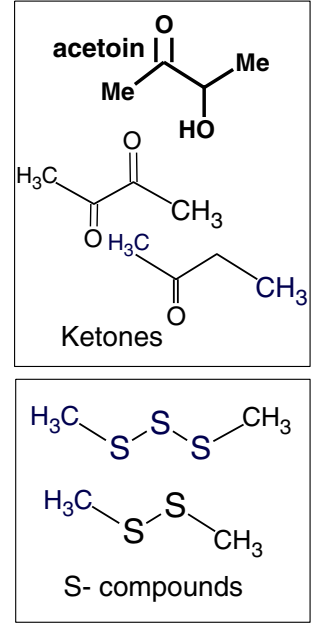

bold, including 2,3-butanediol, acetoin, and tridecane, represent biologically active VOCs that trigger secondary responses in planta and are discussed in the review

ISR when inoculated onto crop seeds in soil failed to induce resistance in the I-plate test. These results suggest that VOCmediated ISR elicitation is not a common mechanism among all PGPR in the rhizosphere.

Which Bacterial Odor is Critical? The next logical step in this investigation was to determine which bacterial VOC(s) play critical roles in ISR. We, therefore, performed gas chromatography combined with mass spectrometry (GC/MS) analysis of the headspace VOCs collected from PGPR strains, which is similar to our previous analysis of plant growth promotion by bacterial VOCs. Analysis for $24 \mathrm{~h}$ revealed the consistent release of 2,3-butanediol and its precursor 3-hydroxy-2butanone from ISR-triggering strains GB03 and IN937a, whereas these VOCs were not released from the non-ISRactivating bacterial strains DH5 $\alpha$ and 89B61 (Ryu et al. 2004a). In fact, most bacterial species of the Proteobacteria and Firmicute groups produce 2,3-butanediol and acetoin under low-oxygen conditions, thus providing an alternative electron sink for the regeneration of NAD + when aerobic respiration is limited (Ramos et al. 2000; Xiao and Xu 2007). Such a scenario may occur in the low-oxygen environment of the rhizosphere, where PGPR naturally reside. Analyzing the comprehensive chemical profile of PGPR volatiles further revealed that a mixture of more than 30 different volatiles are emitted from cultures of Bacillus spp., based on a more advanced headspace solid phase microextraction technique coupled with software extraction of overlapping gas chromatographic (GC)-separated components (Farag et al. 2006; Lee et al. 2012). Further studies should focus on detecting ISR-triggering molecules in this complex blend that are potentially more effective than 2,3butanediol and acetoin. The absolute configuration of GB03synthesized 2,3-butanediol is exclusively in the $(2 R, 3 R)$-form, as determined by chiral GC column chromatography (Ryu et al. GB03 and IN937a, whereas four other PGPR strains that cause 


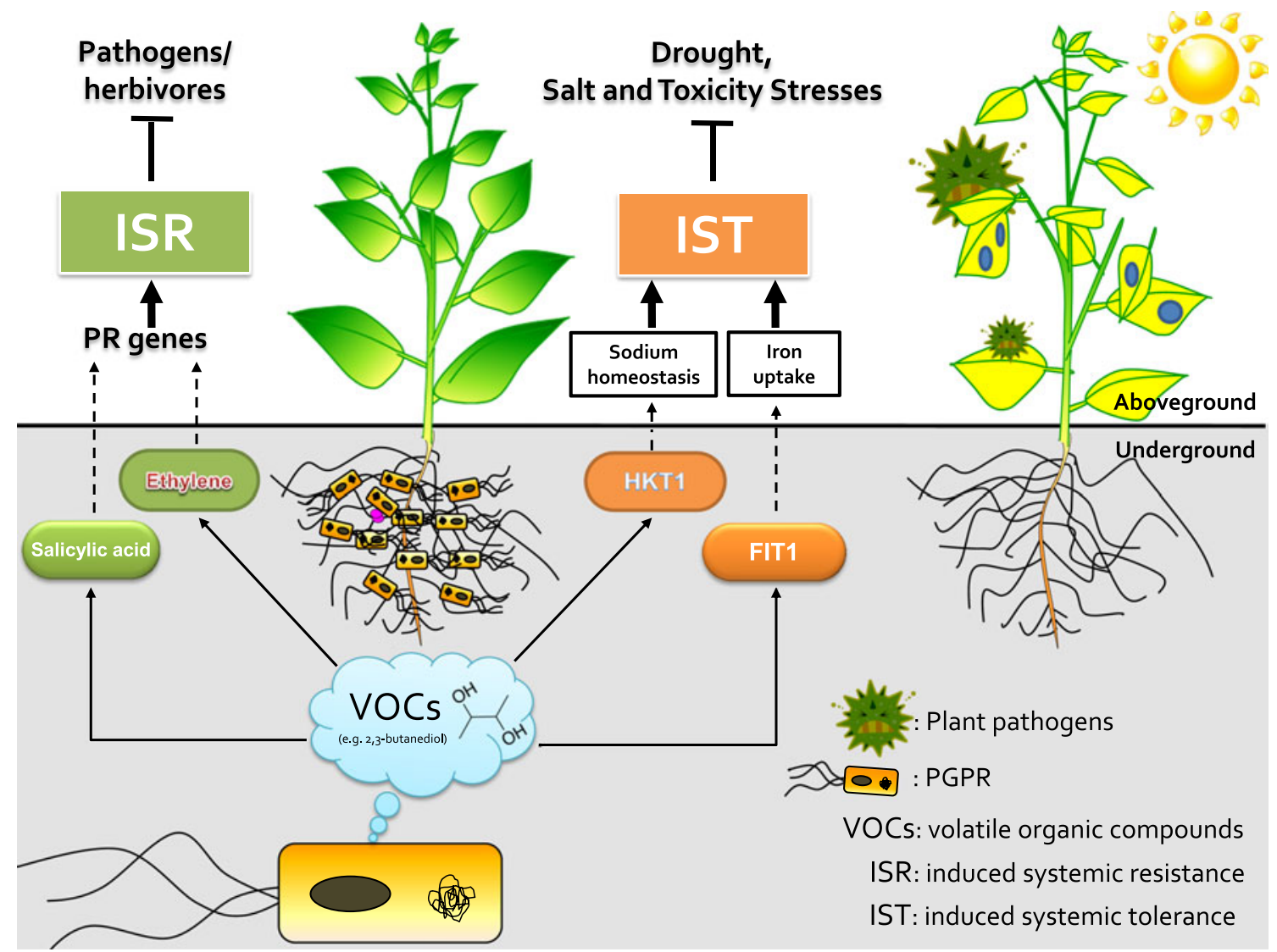

Fig. 3 Model of induced systemic resistance (ISR) and induced systemic tolerance (IST) mechanisms elicited by volatile organic compounds (VOCs) emitted from plant growth-promoting rhizobacteria. ISR and IST elicited by plant growth-promoting rhizobacteria (PGPR) against biotic and abiotic stresses respectively underground (root) and aboveground (shoot). Broken arrows indicate plant responses through individual regulatory component in plants; solid arrows indicate plant compounds affected by bacterial VOCs. Some PGPR strains, indicated in yellowish rods on the plant roots, produce VOCs such as 2,3-butanediol, which results in upregulated pathogenesis-related (PR) genes via salicylic acid and ethylene signalling pathways conferring ISR against phytopathogens and herbivores. Bacterial VOCs downregulate HKT1 expression in roots but upregulate it in shoot tissues, orchestrating lower $\mathrm{Na}+$ levels and recirculation of $\mathrm{Na}+$ and upregulate FIT1 in the whole plant under high salt, metal toxicity, and drought conditions. Abbreviations: FIT1, Fedeficiency-induced transcription factor 1; HKT1, high-affinity $\mathrm{K}+$ transporter 1; ISR, induced systemic resistance; IST, induced systemic tolerance; PGPR, plant-growth-promoting rhizobacteria 2004a). Comparing the bioactivity of both $(2 R, 3 R)$ and $(2 S, 2 S)$ stereoisomers also may help reveal whether stereoisomer specificity exists in PGPR volatiles and whether this specificity is responsible for ISR induction and/or growth promotion in planta. Structural specificity of insect-derived elicitors is evident in the herbivore elicitor volicitin; the L-isomer of this compound is active in VOC emissions, whereas the D-isomer is inactive (Truitt et al. 2004). Pharmaceutical application of synthetic 2,3-butanediol at different doses and a volatile extract collected from strain GB03 show similar disease-protection capacity, which is comparable to that induced by direct PGPR inoculation (Ryu et al. 2004a). Notably, doses of $0.2 \mathrm{pg}$ to $0.2 \mu \mathrm{g} / \mathrm{ml}$ (in increments of 1:100 dilutions) trigger similar levels of ISR, whereas pre-exposure of plants to $20 \mu \mathrm{g}$ of this compound fails to induce ISR.

Recent data from another PGPR strain, P. polymyxa E681, reveal that long-chain bacterial VOCs, i.e., the $\mathrm{C} 13$ hydrocarbon tridecane, also can elicit ISR, as can C4 alcohols such as 2,3butanediol (Lee et al. 2012). The genus Paenibacillus was reclassified as a separate genus from Bacillus due to its distinct biochemical and genetic characteristics (Ash et al. 1993). Nevertheless, Paenibacillus spp. from soil also has beneficial effects on several crop species (McSpadden Gardener 2004). Paenibacillus polymyxa strain E681 from barley root is a promising biocontrol agent that can protect cucumber and sesame from damping-off caused by the soilborne pathogens Fusarium oxysporum, Rhizoctonia solani, and Pythium ultimum (Ryu et al. 2005a, b, 2006). In addition to E681, which has a direct antagonistic effect on fungal growth by producing fungistatic compounds, a novel class of VOC-mediated ISR has been identified (Lee et al. 2012). In that study, ISR against the biotrophic pathogen Pseudomonas syringae was examined, as strain GB03 was previously shown to elicit ISR against a necrotrophic pathogen. 
What Happens in the Plant after the Bacterial "Scent" is Detected? An easy way to dissect plant signaling pathways likely to mediate for the effects of PGPR VOCs in planta is to screen defined signaling pathway mutants and transgenic plant lines following exposure to bacterial volatiles (Han et al. 2006; Ramos et al. 2000; Ryu et al. 2003). Indeed, to elucidate signaling pathway(s) that relate to ISR, a series of mutant and transgenic plant lines were exposed to PGPR VOCs found to trigger ISR through volatile emissions. ISR triggered by GB03 emissions is independent of the salicylic acid, NPR1, and jasmonic acid signaling pathways, although this response appears to be mediated via ethylene. However, ISR activation by strain IN937a is independent of all signaling pathways that were tested, which opens up the possibility that additional VOCs utilize alternative pathways in planta to trigger ISR (Ryu et al. 2004a). These results also were confirmed by using transgenic plant lines containing $\beta$ glucuronidase (GUS) fusions to $P D F 1.2$, a gene induced by JA/ET, as well as by qRT-PCR analysis (Ryu et al. 2004a).. A recent proteomics study that employed Arabidopsis tissue exposed to GBO3 has provided a more detailed picture of how plants perceive PGPR VOCs (Kwon et al. 2010). Of the 95 spots representing proteins showing differential responses to GB03 VOC treatment (including 61 up- and 34 downregulated proteins), 20 spots corresponded to 12 proteins involved in ethylene (ET) biosynthesis. Five representative ET biosynthesis-related genes, namely MAT3, SAM-2, ACS4, $A C S 12$, and $A C O 2$, were significantly up-regulated by this treatment. In addition, the ET marker genes ERF1, GST2, and $C H I B$ were strongly induced by GB03 VOC exposure. Another proteome study that explored the effects of bacterial volatiles effect in planta confirmed that ISR elicitation against $P$. syringae pv. tomato DC3000 by B. subtilis FB17 is mediated via the SA and ET signaling pathways and is independent of the jasmonic acid (JA) pathway (Rudrappa et al. 2010). Bacillus subtilis C4-alcohol biosynthetic pathway mutants fail to elicit ISR, which confirms that acetoin and 2,3-butanediol serve as ISR volatile determinants. Like B. subtilis strain GB03, strain FB17 also was shown to up-regulate $P D F 1.2$ gene transcription. However, how plants modulate and perceive PGPR VOC-elicited defense immediately after pathogen infection remains to be fully elucidated.

Priming the defense pathways by external signals enables the potentiated induction of defense response without immediately activating the defense signaling cascades, which would be accompanied by the expenditure of energy for defense mobilization (Paré et al. 2005). In the case of PGPR priming of plant defenses, induction of the primed state is thought to result in an increase in the amount or activity of cellular components that play important roles in defense signaling; this process is not associated with direct changes in gene expression in leaves (Lee et al. 2012). The priming activity of 2,3-butanediol, thus reducing plant susceptibility to disease, was confirmed in a study that employed altered Bacillus spp. strains that were no longer able to produce this VOC (Ryu et al. 2004a). In a separate study, Han et al. (2006) reported that the application of 2,3-butanediol failed to elicit ISR against Pseudomonas syringae pv. tabaci but did induce the ISR response against $P$. carotovora subsp. carotovora, thus suggesting that different defensive cascades are elicited in response to different pathogens. Indeed, the ethylene (ET)dependent plant defense signaling pathway is more effective against a necrotrophic pathogen such as $P$. carotovorrum subsp. carotovorum than against $P$. syringae, which requires an SA-dependent resistance response (Pieterse et al. 2009).

Long-chain VOC emissions, such as tridecane, released from $P$. polymyxa E681 prime the transcriptional expression of the salicylic acid, jasmonic acid, and ethylene signaling marker genes PR1, ChiB, and VSP2, respectively. Comparison of the signal transduction cascades activated by individual VOCs in the presence or absence of external priming agents should provide more insight into the roles of elicitors and priming agents in triggering plant defense responses and in increasing the long-term fitness of plants (Lee et al. 2012). We hypothesize that the bacterial production of diverse VOCs may help plants modulate defense signaling pathways, including SA and ET signaling, thus protecting them from various types of pathogens, i.e., necrotrophs and biotrophs (Fig. 3).

\section{Induced Systemic Tolerance by Bacterial Volatiles}

As illustrated above, certain PGPR strains can induce changes in plant physiology and signaling when potential pathogens threaten to attack the plant (Pieterse et al. 2002; van Loon et al. 1998). Recently, an increasing number of studies have demonstrated that PGPR and their products, including VOCs, trigger plant tolerance to abiotic stresses, including drought stress, salt stress, and/or nutrient deficiency (Yang et al. 2009). We previously proposed the term induced systemic tolerance (IST) for "PGPR-induced physical and chemical changes in plants that result in enhanced tolerance to abiotic stress". Biotic stress is excluded from IST because conceptually it is a part of biological control and induced resistance. (Yang et al. 2009).

Bacterial VOCs Help Plants Increase Iron Uptake The integrated regulation of orchestrated biological processes, including whole-plant auxin redistribution, leaf cell expansion, root branching, and augmented photosynthesis, underlie plant growth promotion triggered by VOCs from B. subtilis GB03 (Zhang et al. 2007, 2008a). Concomitant with the higher photosynthetic capacity in GB03 VOC-treated plant, increased iron uptake also was observed in these Arabidopsis plants 
(Zhang et al. 2009). Iron is a micronutrient that is essential for the photosynthetic apparatus, due to the redox potential of $\mathrm{Fe}^{2+} / \mathrm{Fe}^{3+}$. In soil, iron is present mainly as insoluble oxyhydroxide polymers, which are not readily taken up by plants. As a result, iron deficiency can occur, especially in plants grown in calcareous soils, which accounts for approximately one-third of the earth's soils (Guerinot and Yi 1994). Under normal growth conditions, GB03 VOCs increase mRNA levels of the Fe-deficiency-induced transcription factor 1 (FIT1) and two of its target genes, ferric reductase $\mathrm{FRO} 2$ and the iron transporter gene IRTI. On the other hand, VOC-triggered enhancement of iron assimilation and photosynthetic efficiency are both compromised in the Arabidopsis fit 1-2 knockout mutant, indicating that FIT1 plays a key role in mediating VOCinduced iron uptake. In addition to the transcriptional regulation of plant iron uptake genes, bacterial volatiles also produce acidification of plant growth media (Zhang et al. 2009), which increases iron mobility in plants (Marschner and Römheld 1994). In fact, rhizosphere acidification also increases the ferric reductase activity of FRO2 (Zhang and Pare, unpublished data). GB03 VOCs lead to rhizosphere acidification via two mechanisms, i.e., elevated proton exudation from roots and direct acidification by unknown VOC component(s). As rhizosphere acidification appears to be an efficient way to increase the uptake of iron (and possibly other nutrients) by plants, experiments should be performed to determine if acidic components are commonly present in volatiles of beneficial soil bacteria and whether acidification-enhanced iron uptake can also be induced by VOCs from other bacteria (Fig. 3).

Modulating Sodium Homeostasis Arabidopsis plants exposed to GB03 VOCs are less susceptible to salt stress than control plants, as the VOC-exposed plants accumulate less $\mathrm{Na}^{+}$in both shoots and roots (Zhang et al. 2008b). The sodium transporter AtHKT1 may function as a pivotal component in mediating VOC-induced salt tolerance. AtHKT1 functions in the removal of $\mathrm{Na}^{+}$from xylem sap. Therefore, the presence of AtHKT1 in roots restricts the uploading of $\mathrm{Na}^{+}$to aerial portions of the plant, whereas in shoots this protein mediates $\mathrm{Na}^{+}$exclusion from the leaves (Horie et al. 2009; Møller et al. 2009). In addition, increasing evidence has shown that AtHKT1 confers shoot-to-root $\mathrm{Na}^{+}$ recirculation, possibly by loading $\mathrm{Na}^{+}$into phloem vessels (Berthomieu et al. 2003; Sunarpi et al. 2005). The root-toshoot ratio of $\mathrm{Na}^{+}$levels in VOC-treated plants is greater than that detected in control plants, which is consistent with the canonical role of root AtHKT1 in restricting $\mathrm{Na}^{+}$in the roots. Moreover, increasing the shoot-to-root recirculation of $\mathrm{Na}^{+}$ also can lead to a higher proportion of $\mathrm{Na}^{+}$in the roots, with less $\mathrm{Na}^{+}$in the shoots, as observed in VOC-treated plants. GB03 VOCs concurrently repress and increase AtHKT1 expression in roots and shoots, respectively. This mechanism affirms the role of AtHKT1 in controlling shoot-to-root $\mathrm{Na}^{+}$ recirculation and helps explain VOC-induced salt tolerance (Zhang et al. 2008b).

Nonetheless, these observations indicate that other components in the $\mathrm{Na}^{+}$homeostasis pathway also may contribute to VOC-induced salt tolerance. The calcium signaling sensor AtSOS3 is required for post-transcriptional activation of the $\mathrm{H}^{+} / \mathrm{Na}^{+}$antiporter AtSOS1, which controls root $\mathrm{Na}^{+}$exudation as well as long-distance $\mathrm{Na}^{+}$transport within plants (Shi et al. 2000). GB03 VOCs triggers an approximately $50 \%$ reduction in whole-plant $\mathrm{Na}^{+}$levels in wild-type plants. By contrast, the Arabidopsis sos 3 mutant exhibits a reduction in $\mathrm{Na}^{+}$ accumulation of only approximately $15 \%$ (Zhang et al. 2008b), suggesting that AtSOS3-dependent $\mathrm{Na}^{+}$exudation is required, at least in part, for the decreased accumulation of $\mathrm{Na}^{+}$ in VOC-treated plants. Intriguingly, VOC treatment does not reduce $\mathrm{Na}^{+}$accumulation in the athktl mutant, indicating that VOC-induced plant salt tolerance is likely an outcome of the integrated regulation of $\mathrm{Na}^{+}$homeostasis. Questions remain about the mechanisms underlying the regulation of $\mathrm{Na}^{+}$ homeostasis, including the following: 1) are abscisic acid (ABA) levels in salt-stressed plants affected by GB03 VOCs, as was observed with un-stressed plants (Zhang et al. 2008a)? 2 ) is the differential regulation of HKT1 gene expression in VOC-treated Arabidopsis dependent on ABI4, which is a transcriptional repressor of HKT1 (Shkolnik-Inbar et al. 2013)? and 3) do GB03 VOCs trigger post-transcriptional regulation of components in the salt stress response pathway? Another intriguing question is whether rhizosphere acidification creates a proton gradient that favors transport of $\mathrm{H}^{+}$into roots, thereby facilitating AtSOS1-dependent $\mathrm{Na}^{+}$exudation (Fig. 3).

Helping Plants Withstand "Thirst" via Bacterial VOCs Arabidopsis plants grown with GB03 or another rhizobacterium, $P$. chlororaphis $\mathrm{O}$, in the soil exhibit increased drought tolerance (Cho et al. 2008; Zhang et al. 2010). Combined GB03 VOCs up-regulate the transcript levels of PEAMT in plants, leading to increased biosynthesis of choline and glycine betaine, two compatible solutes that protect cells from osmotic stress under dehydrating conditions. This enhanced osmo-protection is not caused by alterations in ABA production, as osmotic-stressed plants exhibit VOC-independent accumulation of ABA in both shoots and roots (Zhang et al. 2010). The active components in GB03 VOCs that confer plant stress tolerance have not yet been identified, whereas 2R,3R-butanediol has been shown to be necessary and sufficient for eliciting plant drought tolerance conferred by Pseudomonas chlororaphis O6 (Cho et al. 2008). The SA signaling pathway may be involved in $P$. chlororaphis O6-induced drought tolerance, as droughtstressed plants exposed to either bacterial "scent" or 2,3butanediol accumulate higher levels of SA than untreated plants (Cho et al. 2008). 
Beneficial but Not Almighty Although VOC emissions from rhizobacteria such as $B$. subtilis GB03 have multiple beneficial effects on plants, the same blend of volatiles also may have an adverse effect on plant growth under certain circumstances. For example, it is not known whether the activation of the iron uptake pathway (Zhang et al. 2009) may result in stress phenotypes in plants grown in the presence of toxic heavy metals such as $\mathrm{Cd}$, which employs the same transporter as Fe. In fact, instead of increasing the level of photosynthesis, GB03 VOCs significantly reduce chlorophyll content and photosynthetic efficiency in plants treated with $10 \mu \mathrm{M}$ ABA (Zhang et al. 2008a). Indeed, it is unclear whether a trade-off scenario exists in plants when growth promotion is triggered by bacterial VOCs. A comprehensive review of bacterial VOCs negative impacts on plant growth has been compiled by Bailly and Weisskopf (2012).

\section{The Chemical Side of Bacterial Volatiles}

Decoding the Gaseous Dialog Both solid-phase microextraction (SPME) and dynamic headspace volatile analyses have revealed that active PGPR strains have the requisite machinery to synthesize a wide range of volatiles, including short-chain aliphatic aldehydes, esters, alcohols, organic acids, ethers, ketones, sulfur compounds, and hydrocarbons (Fig. 2). Among the PGPR strains that were examined, $B$. amyloliquefaciens IN937a produces the largest volume of VOCs, followed by B. subtilis GB03 (Farag et al. 2006). Alcohols represent the most abundant compounds consistently released from GB03 and IN937a strains, with 2,3-butanediol and 3-hydroxy-2-butanone (also referred to as acetoin) identified as bioactive molecules that trigger both growth promotion and ISR (Ryu et al. 2003, 2004a). Oxidized and methylated products of these fusel alcohols were identified in later studies, including aldehydes, ethers, and acids, all of which have yet to be tested for possible biological effects (Farag et al. 2006). In Bacillus sp., 2,3-butanediol and acetoin are products of an alternative reductive pathway originating from pyruvate. This pathway produces an alternative source of $\mathrm{NAD}^{+}$under anaerobic conditions and is analogous to alcohol fermentation, which is activated in yeast under low atmospheric $\mathrm{O}_{2}$. By contrast, the accumulation of the branched alcohols 3-methyl-1butanol, 2-methyl-1-butanol, and 2-methyl-1-propanol in Bacillus amyloliquefaciens IN937a headspace volatiles suggests that these compounds are formed as degradation products of the branched amino acids leucine, isoleucine, and valine, respectively, via the Ehrlich pathway, as in yeast (Dickinson et al. 1997; Marilley and Casey 2004). This pathway involves the concerted actions of a transaminase, a decarboxylase, and an alcohol dehydrogenase, as is also the case in yeast and lactic acid bacteria. Catabolic products of the sulfur-containing amino acids methionine and cysteine also were detected, as exemplified by dimethyldisulfide (DMDS) and dimethyl trisulfide (DMTS), which are found in most PGPR strains and in lactic acid bacteria (Seefeldt and Weimer 2000) and fungi. We still do not have a clear picture of the biological or ecological relevance of the latter compounds in PGPR. The biogenetic origins of many of these compounds have been determined in other organisms but not in PGPR; the in vivo functions of these branched alcohols in PGPR also have not been fully elucidated. Their origins can be unambiguously determined by feeding precursor isotopes to PGPR cultures and monitoring for label enrichment in the released volatiles. Volatile compounds can be extracted directly from bacterial culture supernatants, and their ${ }^{13} \mathrm{C}$ enrichment levels can be obtained by GC coupled with isotope ratio mass spectrometry (IRMS) (Goupry et al. 2000).

Recent data from VOCs profiling studies in $P$. polymyxa E681 show that the long-chain $\mathrm{C} 13$ bacterial volatile tridecane augments ISR by defense priming (Lee et al. 2012). To date, the microbial production of this hydrocarbon has not been studied extensively. The observed variation in volatile profiles among various PGPR strains suggests that diverse VOC metabolic mechanisms exists among PGPR, and supports the idea that VOCs can serve as taxonomic markers in PGPR, as in other microbial systems (Kim et al. 2013; Scholler et al. 2002). To further analyze differences in volatile production among strains in an untargeted manner, chemometric data analysis, as exemplified by principal component analysis (PCA), could be performed on VOC quantification data. Coupling the differential VOC profiles from different PGPR strains with the analysis of differential levels of gene transcription may be useful for probing biosynthetic pathways leading to volatile production in PGPR strains. Pharmacological doses of 2,3butanediol and tridecane tested in (Ryu et al. 2004a) and (Lee et al. 2012), respectively, were comparable to those released from PGPR grown in vitro on Murashige-Skoog (MS) media and later from PGPR inoculated on potato root under low oxygen pressure (Farag et al. 2006), but have yet to be compared with VOCs levels produced in the soil. By growing PGPR and Arabidopsis seedlings on separate sides of divided petri dishes, only were we able to examine the role of airborne bacterial metabolites in triggering ISR. Nevertheless, we note that a possible scenario that volatiles produced from PGPR residing on the plant root can act directly and elicit an effect is supported by the detection of 2,3-butanediol from PGPR inocculated on potato root (Farag et al. 2006). Detection of these bioactive volatiles from PGPR grown on plant roots in the rhizosphere, and whether it can diffuse within soil particles at sufficient levels to elicit response in neighboring roots, is a topic that has yet to be investigated.

Technical Advances in the Characterization of PGPR Volatiles A variety of systems have been developed to capture, analyze, identify, and quantify airborne volatiles released from plants. Methodology commonly used for airborne volatile 
analysis is based on headspace analysis followed by GC analysis (Fig. 4). Headspace volatiles can be extracted from a dynamic air flow over a bacterial culture onto an absorbent filter and released by rinsing the filter with organic solvent. Conversely, the volatiles can be collected in the absence of air flow by SPME and directly released into a heated GC injector. For an in depth review of volatiles collection methods (Tholl et al. 2006) should be consulted. The number of detectable volatiles in an organism blend generally increases when various techniques are applied, e.g., dynamic headspace volatile capture in open and closed airflow systems, different trapping materials, SPME, GC/MS, and proton transfer reaction/mass spectrometry (PTR-MS), as summarized in Wenke et al. (2012). Indeed, no single analytical method can accurately survey the entire volatiles profile of a living organism i.e., plants or bacteria. However, the combination of several sampling techniques is a powerful methodology for identifying of volatiles. Because of the complementary analytical features of static closed SPME volatiles sampling technique versus dynamic headspace sampling, opportunities for leveraging both methods are being considered, which could create more comprehensive volatiles profiling. In addition, concerns about chemical bias using one technique can be circumvented through a combination of both methods to help provide a more complete and accurate profile of volatiles released from a sample under investigation.

Unlike airborne VOCs such as plant volatiles, which can easily be sampled by headspace collections of living plants, rhizosphere emissions by PGPR present the complication of requiring the desorption of low molecular weight compounds from the soil matrix. By growing PGPR in Petri dishes (Ryu et al. 2003, 2004a), we were able to examine and identify the role of airborne bacterial metabolites in triggering ISR and growth promotion. To date, two different types of headspace sampling techniques have been employed to investigate PGPR volatiles.

The first analysis was performed using dynamic headspace sampling, in which headspace volatiles were extracted from a dynamic continuous humidified air flow over PGPR grown on solid Murashige-Skoog (MS) medium onto divinyl benzene absorbent filters (Super Q, Alltech) and then released by rinsing with methylene chloride (Ryu et al. 2003, 2004a). We determined that MS medium produces lower background signals than other media such as LuriaBertani media (LB); the peptone in these media releases a large number of volatiles, which interferes with the analysis of volatiles derived from PGPR strains (unpublished data). Furthermore, the effects of growth media and conditions on the PGPR emission spectra have to be considered (Ryu et al. 2003, 2004a).

A review by (Bailly and Weisskopf 2012) into the culture conditions for Bacillus species within PGPR revealed that promoting effects was observed when Murachige and Skoog media was used to grow the bacteria as well as the plants. Albeit, when LB [or the similar medium nutrient broth agar (NA)] were used, the volatile-mediated effect was abolished, if not an inhibitory effect observed, which reveals the major effect of media composition on PGPR released VOCs. Enrichment of C-source (i.e., sucrose) in MS medium versus
Fig. 4 Proposed scheme for volatile analysis procedure from plant growth-promoting rhizobacteria (PGPR) showing the following steps: I) volatile collection using either a dynamic headspace sampling by blowing humidified air over PGPR cultures and venting through adsorbent filters, or b static solid phase microextraction (SPME) using fibers, later desorbed inside GC, followed by II) volatile analysis using GC/MS and peaks deconvolution using AMDIS software, allowing for the resolution of complex volatile blend components, III) multivariate data analysis i.e., principal component analysis (PCA) to help reveal differences and similarities between PGPR strains

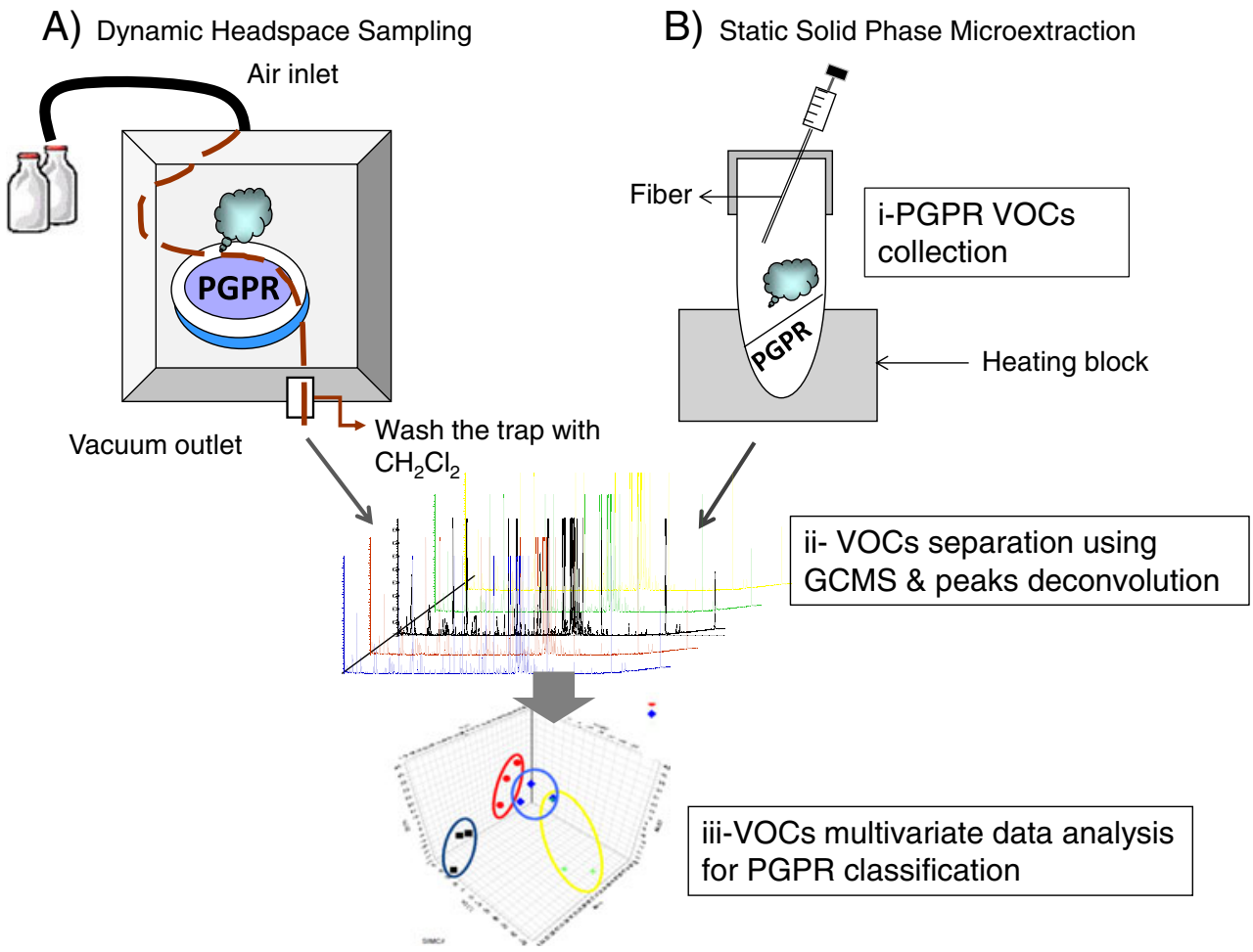


nitrogen (i.e., proteins) in LB is likely to account for VOC variation. The impact of the cultivation medium on the volatiles mediated effect of bacteria on plants was also assessed by (Blom et al. 2011) using a collection of Burkholderia strains and affirming effect of media on volatiles composition and effect on plants.

Results from the studies by Ryu et al. (2003, 2004a) successfully led to the identification of the two bioactive volatile chemicals, acetoin and 2,3 butanediol, from the GB03 and IN937a strains. Nevertheless, the continuous air flow over bacterial cultures in the volatile setup employed (Ryu et al. 2003, 2004a) may not closely mimic the low oxygen partial pressures usually present in the root environment where PGPR naturally reside (Kloepper et al. 1999). In addition, the low adsorption affinity of Super Q filters for low molecular weight metabolites may have reduced collection yields.

To more thoroughly examine specific differences in VOC emission profiles, headspace SPME, coupled with software extraction of overlapping GC-separated components, was employed (Fig. 4). SPME can extract volatiles from bacterial cultures in a relatively short amount of time, usually $<30 \mathrm{~min}$ in a no-flow, low oxygen environment, and has been successfully used to collect them in several systems (Goupry et al. 2000; Marilley and Casey 2004). Despite the advantages of SPME, fiber coatings need to be considered with caution, as they can limit sensitivity by preferentially absorbing or excluding particular analytes based on polarity or size. For example, PDMS fiber preferentially adsorbs nonpolar metabolites, whereas divnylbenzene/carboxen/PDMS (DCP) fiber favors short-chain polar compounds (Doleschall et al. 2003; Jelen et al. 2000). In the case of PGPR volatiles, divnylbenzene/carboxen/PDMS fiber provides the best recovery of VOCs; it favors the adsorption of polar low molecular weight VOCs, which are the predominant VOCs released from PGPR strains. A total of 28 new volatile components that were not identified by dynamic headspace sampling were identified from PGPR strains (Farag et al. 2006) using SPME, with more differences in VOCs revealed among PGPR strains. The increase in the identification of VOCs was due, in part, to the increased sensitivity of the fiber towards low molecular weight volatiles. In addition, processing the MS files with AMDIS software assisted in adjacent peak deconvolution and background subtraction and increased the detection limit (Farag and Wessjohann 2012; Halket et al. 1999). Indeed, the application of metabolomic identification strategies for volatile analysis has great potential for examining PGPR, as the small molecules released from bacteria are highly polar, with a strong tendency to co-elute (Fig. 4). This factor can lead to the production of overlapping MS spectra, which hinders accurate identification of volatiles and affects peak quality matching against chemical databases (Farag and Wessjohann 2012). A variety of resources can be used to identify unknown compounds in a given volatile sample, including $>600,000$ compounds with known mass spectra cataloged in searchable mass spectral libraries and/or kovat index (KI) measurements. In future analyses of PGPR VOCs, perhaps in situ volatile collection methods will be developed to determine whether bioactive PGPR volatiles are released in the rhizosphere where PGPR normally reside and whether ISR and the growth promotion effect elicited by PGPR VOCs can occur in soil or soil-less medium. Measuring emissions of volatile isoprenoids from roots in vivo was made feasible under natural field conditions by using a dynamic bag enclosure method developed by (Lin et al. 2007), which could be considered for monitoring PGPR VOCs in the rhizosphere. Nevertheless the preparation method used for dynamic enclosure sampling was found to affect magnitude and composition of emissions which has to be adapted in the case of PGPR.

\section{Applications and Perspectives}

Field Application of Bacterial Volatile(s) Despite their volatile nature, VOCs may have practical applications, as many VOCs, such as 2,3-butanediol, are water soluble, inexpensive $(<\$ 1 / \mathrm{kg})$, function at extremely low concentrations (at the $\mathrm{ng}-\mathrm{pg} / \mathrm{ml}$ level), and appear to be safe to animals and humans. Under growth chamber conditions, the direct application of acetoin to roots produces a significant reduction in pathogen growth at $96 \mathrm{hr}$ post challenge (Rudrappa et al. 2010). Therefore, bacterial VOCs represent good candidates for improving disease control through the enhanced management of induced disease resistance. Nevertheless, the primary challenge to field application of bacteria-derived volatiles is developing adequate methods of chemical treatment. These volatiles are released at very low, steady levels from bacteria, a process that is difficult to mimic. Indeed, the application of a single, high dose of a VOC may stress a plant, as observed after the application of other volatile signaling molecules such as MeJA (Kessler and Bladwin 2001). The high vapor pressure of these short-chain volatiles, with low boiling points, also could lead to significant losses of compounds upon application. A controlled-release form of PGPR VOCs, as pioneered for the application of phytohormones (Kessler and Bladwin 2001), has yet to be developed in the case of PGPR. PGPR volatiles have other drawbacks as well, including the high evaporation rate after application in the open field, their negative effects on plant growth, and their inconsistent levels of effectiveness.

Another method for applying VOCs to plants involves drench application of bacterial volatiles. This method has been successfully applied to pepper roots and seeds as well as cucumber seeds, leading to defense priming and ISR under field conditions for four consecutive years (Ryu laboratory, unpublished data). In addition, 4-week-old pepper plants 
were dip-treated with $1 \mathrm{mM} 3$-pentanol solution before they were transplanted to the field. This process elicited ISR in 2year field trials without affecting fruit yield. Drench application of the volatiles 3-pentanol and 2-butanone upregulated the defense-related gene $C S L O X$ in cucumber, leading to a decrease in the population of the sucking insect aphid (Myzus persicae) and significantly increasing the population of its natural enemy, ladybird beetle. These results demonstrate that triggering the oxylipin pathway in response to PGPR VOCs can help recruit a natural enemy of aphids and may ultimately prevent plant disease and insect damage by eliciting induced resistance, even under open field conditions (Song and Ryu 2013). Whether PGPR volatiles function as cues that can attract natural enemies of herbivores, or whether these volatiles induce volatile emissions from plants, which has been shown to occur in several agricultural species (Arimura et al. 2000; Farag and Paré 2002), has yet to be determined. Both scenarios are supported by the fact the GB03 volatiles lead to an increase in the accumulation of essential oils and an increase in emissions in sweet basil (Banchio et al. 2009). In addition, C6-volatiles, which share structural homology with C4alcohols released from PGPR, also trigger terpene emission, which is similar to the effects of herbivore damage (Farag and Paré 2002).

Perspectives The treatment of (aerial parts of) plants with highly active but inexpensive compounds, such as 2,3butanediol, for growth promotion, ISR induction, drought tolerance, and salinity tolerance represents a novel, promising agricultural strategy. The Petri dish assays that were employed by Ryu et al. (2003, 2004a) exposed whole plants to plumes of bacterial VOCs. Therefore, it is unknown whether the site of VOC perception for soil-grown plants is above or below ground. The sphere of microbial emissions for rhizosphere bacteria may be within the soil and/or above ground; the possibility exists that VOCs are produced at a sufficient level for aerial tissues to perceive and respond to these substances. An alternative scheme is that an endogenous signal or signals transport information from the root zone to the aerial portion of the plant. The observation that induced resistance is systemic (Pieterse et al. 2009; van Loon et al. 1998) necessitates the presence of some mobile messenger within the plant. In addition, a detailed dissection of bacterial VOC-mediated ISR will be required to understand how plant immune responses are elicited. In particular, the plant defense-boosting system referred to as "defense priming" is an important topic to consider (Conrath et al. 2002).

As is the case for volatiles released and acting above ground on leaves, nothing is known about the site or mechanism of volatile perception. The critical link in establishing the biological significance of PGPR volatiles in triggering plant growth promotion and defense responses is to ascertain whether these components function in the root, where PGPR naturally resides, or whether volatiles are in fact translocated from the root to the shoot in situ. Indeed, it is not yet known whether C4-alcohols such as 2,3-butanediol serve as mobile chemical messengers to trigger responses in other plant portions, or if another mobile signal serves as a chemical messenger. Structurally related, C6-alcohols released in response to insect damage induce systemin (mobile defense polypeptide) production in addition to triggering a systemic indirect defense response in tomato (Farag and Paré 2002; Sivasankar et al. 2000). A similar scenario also may occur in PGPR VOC-plant interactions. Tracking radiolabeled, synthetic 2,3-butanediol applied to the root in other aerial parts of plants may affirm whether this molecule serves as the mobile signal in plants or whether it triggers another mobile signaling cascade. How plants perceive and respond to PGPR may be clarified by using large scale analysis techniques that screen plant mutants, and by monitoring transcriptional/proteomic/ metabolomic changes in response to individual VOC treatments.

Analyzing biological structure-activity relationships between natural and synthetic $\mathrm{C} 4$ alcohols also may help identify more biologically active volatiles and reveal crucial structural motifs in PGPR VOCs that promote growth and/or induce ISR in planta. Structure-activity relationships have been observed among C6-volatiles in triggering defense gene induction in maize (Farag et al. 2005). Another important structural motif for C4-volatile elicitation that needs to be examined is the four-carbon chain length, including whether a reduction or extension of the original C4-unit can deactivate 2,3-butanediol or acetoin, rendering these molecules unable to trigger ISR or promote plant growth.

Acknowledgments This study was supported by grants from the Industrial Source Technology Development Program of the Ministry of Knowledge Economy (10035386) of Korea, the Next-Generation BioGreen 21 Program (SSAC grant \#PJ009524), Rural Development Administration, , and the KRIBB Initiative Program, South Korea, for Dr. Choong-Min Ryu, and The Alexander von Humboldt Foundation, Germany for Dr. Mohamed A. Farag.

Open Access This article is distributed under the terms of the Creative Commons Attribution License which permits any use, distribution, and reproduction in any medium, provided the original author(s) and the source are credited.

\section{References}

Arimura GI, Ozawa R, Shimoda T, Nishioka T, Boland W, Takabayashi $\mathrm{J}$ (2000) Herbivory-induced volatiles elicit defense genes in lima bean leaves. Nature 406:512-515

Ash C, Priest FG, Collins MD (1993) Molecular identification of rRNA group 3 bacilli (Ash, Farrow, Wallbanks and Collins) using a PCR 
probe test. Proposal for the creation of a new genus Paenibacillus. Anton Leeuw. Int J G 64:253-260

Bailly A, Weisskopf L (2012) The modulating effect of bacterial volatiles on plant growth: current knowledge and future challenges. Plant Signal Behav 7:79-85

Banchio E, Xie X, Zhang H, Paré PW (2009) Soil bacteria elevate essential oil accumulation and emissions in sweet basil. J Agric Food Chem 57:653-657

Bate NJ, Rothstein SJ (1998) C6-volatiles derived from the lipoxygenase pathway induce a subset of defense-related genes. Plant J 16:561-569

Berthomieu P, Conéjéro G, Nublat A, Brackenbury WJ, Lambert C, Savio C, Uozumi N, Oiki S, Yamada K, Cellier F, Gosti F, Simonneau T, Essah PA, Tester M, Véry AA, Sentenac H, Casse F (2003) Functional analysis of AtHKT1 in Arabidopsis shows that $\mathrm{Na}(+)$ recirculation by the phloem is crucial for salt tolerance. EMBO J 22:2004-2014

Bleeker AB, Kende H (2000) Ethylene: a gaseous signal molecule in plant. Annu Rev Cell Dev Biol 16:1-18

Blom D, Fabbri C, Connor EC, Schiestl FP, Klauser DR, Boller T, Eberl L, Weisskopf L (2011) Production of plant growth modulating volatiles is widespread among rhizosphere bacteria and strongly depends on culture conditions. Environ Microbiol 13:3047-3058

Cho SM, Kang BR, Han SH, Anderson AJ, Park JY, Lee YH, Cho BH, Yang KY, Ryu C-M, Kim YC (2008) 2R,3R-butanediol, a bacterial volatile produced by Pseudomonas chlororaphis O6, is involved in induction of systemic tolerance to drought in Arabidopsis thaliana. Mol Plant Microbe Interact 21:1067-1075

Conrath U, Pieterse CMJ, Mauch-Mani B (2002) Priming in plantpathogen interactions. Trends Plant Sci 7:210-216

Croft KPC, Juttner F, Slusarenko AJ (1993) Volatile products of the lipoxygenase pathway evolved from Phaseolus-vulgaris (L) leaves inoculated with Pseudomonas syringae pv. phaseolicola. Plant Physiol 101:13-24

Dickinson RJ, Lanterman MM, Danner JD, Pearson BM, Sanz P, Harrison SJ, Hewlins MJE (1997) $\mathrm{A}^{13} \mathrm{C}$ nuclear magnetic resonance investigation of the metabolism of leucine to isoamyl alcohol in Saccharomyces cerevisiae. J Biol Chem 272:26871-26878

Doleschall F, Recseq K, Kemeny Z, Kovari K (2003) Comparison of differently coated SPME fibres applied for monitoring volatile substances in vegetable oils. Eur J Lipid Sci Technol 105:333-338

Effmert U, Kalderás J, Warnke R, Piechulla B (2012) Volatile mediated interactions between bacteria and fungi in the soil. J Chem Ecol 38:665703

Farag MA, Paré PW (2002) C6-Green leaf volatiles trigger local and systemic VOC emissions in tomato. Phytochemistry 61:545-554

Farag MA, Wessjohann LA (2012) Volatiles profiling in medicinal licorice roots using steam distillation and solid-phase microextraction (SPME) coupled to chemometrics. J Food Sci 77:C1179-C1184

Farag MA, Fokar M, Zhang H, Allen R, Paré PW (2005) (Z)-3-hexenol induce defense genes and down-stream metabolites in maize. Planta 220:900-909

Farag MA, Ryu C-M, Sumner LW, Paré PW (2006) GC-MS SPME profiling of rhizobacterial volatiles reveals prospective inducers of growth promotion and induced systemic resistance in plants. Phytochemistry 67:2262-2268

Fidantsef AL, Stout MJ, Thaler JS, Duffey SS, Bostock RM (1999) Signal interactions in pathogen and insect attack: expression of lipoxygenase, proteinase inhibitor II, and pathogenesis-related protein P4 in the tomato, Lycopersicon esculentum. Physiol Mol Plant P 54:97-114

Gardener HW, Dornbos DL, Desjardins A (1990) Hexanal, trans-2hexenal, and trans-2-nonenal inhibit soybean, Glycine max, seed germination. J Agric Food Chem 38:1316-1320

Glick BR (1995) The enhancement of plant growth by free-living bacteria. Can J Microiol 41:109-117
Goupry S, Rochut N, Robins RJ, Gentil E (2000) Evaluation of solid-phase microextraction for the isotopic analysis of volatile compounds produced during fermentation by lactic acid bacteria. J Agric Food Chem 48:2222-2227

Guerinot ML, Yi Y (1994) Iron: Nutritious, noxious, and not readily available. Plant Physiol 104:815-820

Halket JM, Pryzborowska A, Stein SE, Mallard G, Down S, Chalmers RA (1999) Deconvolution gas chromatography/mass spectrometry of urinary organic acids-potential for pattern recognition and automated identification of metabolic disorders. Rapid Commun Mass Spectrom 13:279-284

Han SH, Lee SJ, Moon JH, Park KH, Yang KY, Cho BH, Kim KY, Kim YW, Lee MC, Anderson AJ, Kim YC (2006) GacSdependent production of 2R, 3R-butanediol by Pseudomonas chlororaphis $\mathrm{O} 6$ is a major determinant for eliciting systemic resistance against Erwinia carotovora but not against Pseudomonas syringae pv. tabaci in tobacco. Mol Plant Microbe Interact 19:924-930

Hatanaka A, Kajiwara T, Sekiya J (1987) Biosynthesis pathway for C6aldehydes formation from linolenic acid in green leaves. Chem Phys Lipids 44:341-361

Hildebrand DF, Brown GC, Jackson DM, Hamilton TR (1993) Effect of some leaf emitted volatiles compounds on aphid population increase. J Chem Ecol 19:1875-1887

Horie T, Hauser F, Schroeder JI (2009) HKT transporter-mediated salinity resistance mechanisms in Arabidopsis and monocot crop plants. Trends Plant Sci 14:660-668

Jelen HH, Obuchowska M, Zawirska-Wojtasiak R, Wasowicz E (2000) Headspace solid-phase microextraction use for the characterization of volatile compounds in vegetable oils of different sensory quality. J Agric Food Chem 48:2360-2367

Kessler A, Bladwin IT (2001) Defensive function of herbivore-induced plant volatile emissions in nature. Science 291:2141-2144

Kim KS, Lee S, Ryu CM (2013) Interspecific bacterial sensing by airborne signals leads to modulated locomotion and drugresistance. Nat Commun 4:1809

Kloepper JW (1992) Plant growth-promoting rhizobacteria as biological control agents. In: Metting FB Jr (ed) Soil microbial ecology: Applications in agricultural and environmental management. Marcel Dekker Inc, NY, pp 255-274

Kloepper JW, Rodriguez-Kabana R, Zehnder GW, Murphy J, Sikora E, Fernandez C (1999) Plant root-bacterial interactions in biological control of soilborne diseases and potential extension to systemic and foliar diseases. Australas Plant Pathol 28:27-33

Kloepper JW, Ryu CM, Zhang S (2004) Induced systemic resistance and promotion of plant growth by Bacillus spp. Phytopathology 94:1259 1266

Kwon YS, Ryu CM, Lee S, Park HB, Han KS et al (2010) Proteome analysis of Arabidopsis seedlings exposed to bacterial volatiles. Planta 232:1355-1370

Lee B, Farag MA, Park HB, Kloepper JW, Lee SH, Ryu CM (2012) Induced resistance by a long-chain bacterial volatile: elicitation of plant systemic defense by a $\mathrm{C} 13$ volatile produced by paenibacillus polymyxa. PLoS One 7:e48744

Lin C, Owen S, Penuelas J (2007) Volatile organic compounds in the roots and rhizosphere of Pinus spp. Soil Biol Biochem 39:951-960

Liu L, Kloepper JW, Tuzun S (1995) Induction of systemic resistance in cucumber against bacterial angular leaf spot by plant growthpromoting rhizobacteria. Phytopathology 85:843-847

Marilley L, Casey MG (2004) Flavours of cheese products: metabolic pathways, analytical tools and identification of producing strains. Int J Food Microbiol 90:139-159

Marschner H, Römheld V (1994) Strategies of plants for acquisition of iron. Plant Soil 165:261-274

McSpadden Gardener BE (2004) Ecology of Bacillus and Paenibacillus spp. in agricultural ystems. Phytopathology 9411:1252-1258 
Møller IS, Gilliham M, Jha D, Mayo GM, Roy SJ, Coates JC, Haseloff J, Tester M (2009) Shoot Na+ exclusion and increased salinity tolerance engineered by cell type-specific alteration of $\mathrm{Na}+$ transport in Arabidopsis. Plant Cell 21:2163-2178

Murphy JF, Reddy MS, Ryu CM, Kloepper JW, Li R (2003) Rhizobacteriamediated growth promotion of tomato leads to protection against Cucumber mosaic virus. Phytopathology 93:1301-1307

Paré PW, Tumlinson JH (1999) Plant volatiles as a defense against insect herbivores. Plant Physiol 121:325-331

Paré PW, Farag MA, Zhang H, Ryu CM, Kloepper JW (2005) Elicitors and priming agents initiate plant defense responses. Photosynth Res 85:149-159

Pieterse CMJ, Van Wees SCM, Ton J, Van Pelt JA, Van Loon LC (2002) Signaling in rhizobacteria-induced systemic resistance in Arabidopsis thaliana. Plant Biol 4:535-544

Pieterse CM, Leon-Reyes A, Van der Ent S, Van Wees SC (2009) Networking by small-molecule hormones in plant immunity. Nat Chem Biol 5:308-316

Ramos HC, Hoffmann T, Marino M, Nedjari H, PresecanSiedel E, Dreesen O, Glaser P, Jahn D (2000) Fermentative metabolism of Bacillus subtilis: physiology and regulation of gene expression. J Bacteriol 182:3072-3080

Rudrappa T, Biedrzycki ML, Kunjeti SG, Donofrio NM, Czymmek KJ, Paré PW, Bais HP (2010) The rhizobacterial elicitor acetoin induces systemic resistance in Arabidopsis thaliana. Commun Integr Biol 3:130-138

Ryu CM, Farag MA, Hu CH, Reddy MS, Wei HX, Paré PW, Kloepper JW (2003) Bacterial Volatiles Promote Growth in Arabidopsis. Proc Natl Acad Sci USA 100:4927-4932

Ryu CM, Farag MA, Hu CH, Reddy MS, Kloepper JW, Paré PW (2004a) Bacterial volatiles induce systemic resistance in Arabidopsis. Plant Physiol 134:1017-1026

Ryu CM, Murphy JF, Mysore KS, Kloepper JW (2004b) Plant growthpromoting rhizobacteria protect systemically Arabidopsis thaliana against Cucumber mosaic virus by a salicylic acid and NPR1independent and jasmonic acid dependent signaling pathway. Plant J 39:381-392

Ryu CM, Hu CH, Locy RD, Kloepper JW (2004c) Study of mechanisms for plant growth promotion elicited by rhizobacteria in Arabidopsis thaliana. Plant Soil 268:285-292

Ryu CH, Kim J, Choi O, Park SY, Park SH (2005a) Nature of a rootassociated Paenibacillus polymyxa from field-grown winter barley in Korea. J Microbiol Biotechnol 15:984-991

Ryu CM, Hu CH, Locy RD, Kloepper JW (2005b) Study of mechanisms for plant growth promotion elicited by rhizobacteria in Arabidopsis thaliana. Plant Soil 268:285-292

Ryu CM, Kim J, Choi O, Kim SH, Park CS (2006) Improvement of biological control capacity of Paenibacillus polymyxa E681 by seed pelleting on sesame. Biol Control 39:282-289

Scholler CE, Gurtler H, Pederson R, Molin S, Wilkins K (2002) Volatile metabolites from actinomycetes. J Agric Food Chem 50:2615-2621

Seefeldt KE, Weimer BC (2000) Diversity of sulphur compound production in lactic acid bacteria. J Dairy Sci 83:2740-2746

Shi H, Ishitani M, Kim C, Zhu JK (2000) The Arabidopsis thaliana salt tolerance gene SOS1 encodes a putative $\mathrm{Na}+\mathrm{H}+$ antiporter. Proc Natl Acad Sci USA 97:6896-6901

Shkolnik-Inbar D, Adler G, Bar-Zvi D (2013) ABI4 downregulates expression of the sodium transporter HKT1;1 in Arabidopsis roots and affects salt tolerance. Plant J 73:993-1005
Shulaev V, Silverman P, Raskin I (1997) Airborne signalling by methyl salicylate in plant pathogen resistance. Nature 385:718-721

Sivasankar S, Sheldrick B, Rothstein SJ (2000) Expression of allene oxide synthase determines defense gene activation in tomato. Plant Physiol 122:1335-1342

Song GC, Ryu C-M (2013) Two volatile organic compounds trigger plant self-defense against a bacterial pathogen and a sucking insect in cucumber under open field conditions. Int J Mol Sci 14:9803-9819

Sunarpi, Horie T, Motoda J, Kubo M, Yang H, Yoda K, Horie R, Chan WY, Leung HY, Hattori K, Konomi M, Osumi M, Yamagami M, Schroeder JI, Uozumi N (2005) Enhanced salt tolerance mediated by AtHKT1 transporter-induced Na unloading from xylem vessels to xylem parenchyma cells. Plant J 44:928-938

Tholl D, Boland W, Hansel A, Loreto F, Ursula SR, Röse USR, Schnitzler J-P (2006) Practical approaches to plant volatile analysis. Plant J 45:540-560

Truitt CL, Wei HX, Paré PW (2004) A plasma membrane protein from Zea mays binds with the herbivore elicitor volicitin. Plant Cell 16:523-532

Van Loon LC, Bakker PAHM, Pierterse CMJ (1998) Systemic resistance induced by rhizosphere bacteria. Annu Rev Phytopath 36:453-483

Weidhase RA, Kramell HM, Lehmann J, Leibisch HW, Lerbs W, Parthier B (1987) Methyl jasmonate-induced changes in the polypeptide pattern of senescing barley leaf segments. Plant Sci 51:177-186

Wenke K, Kai M, Piechulla B (2010) Belowground volatiles facilitate interactions between plant roots and soil organisms. Planta 231:499-506

Wenke K, Weise T, Warnke R, Valverde C, Wanke D, Kai M, Piechulla B (2012) Bactertial volatiles mediating information between bacteria and plants. In: Biocommunication of plants. Springer Berlin Heidelberg. 14:327-347

Xiao ZJ, Xu P (2007) Acetoin metabolism in bacteria. Crit Rev Microbiol 33:127-140

Yang J, Kloepper JW, Ryu CM (2009) Rhizosphere bacteria help plants tolerate abiotic stress. Trends Plant Sci 14:1-4

Zehnder GW, Yao C, Murphy JF, Sikora ER, Kloepper JW, Schuster DJ, Polston JE (1999) Microbe-induced resistance against pathogens and herbivores: Evidence of effectiveness in agriculture. Induced plant defenses against pathogens and herbivores: biochemistry, ecology and agriculture. APS. St. Paul. MN. 33:182

Zeringue HJ (1992) Effects of C6-C10 alkenals and alkanals on eliciting a defense response in the developing cotton ball. Phytochemistry 3:2305-2308

Zhang H, Kim MS, Krishnamachari V, Payton P, Sun Y, Grimson M, Farag MA, Ryu CM, Allen R, Melo IS, Paré PW (2007) Rhizobacterial volatile emissions regulate auxin homeostasis and cell expansion in Arabidopsis. Planta 226:839-851

Zhang H, Kim MS, Sun Y, Dowd SE, Shi H, Paré PW (2008a) Soil bacteria confer plant salt tolerance by tissue-specific regulation of the sodium transporter HKT1. Mol Plant Microbe In 21:737-744

Zhang H, Xie X, Kim MS, Kornyeyev DA, Holaday S, Paré PW (2008b) Soil bacteria augment Arabidopsis photosynthesis by decreasing glucose sensing and abscisic acid levels in planta. Plant J 56:264-273

Zhang H, Sun Y, Xie X, Kim MS, Dowd SE, Paré PW (2009) A soil bacteria regulates plant acquisition of iron via deficiency-inducible mechanisms. Plant J 58:568-577

Zhang H, Murzello C, Sun Y, Kim MS, Xie X, Jeter RM, Zak JC, Dowd SE, Paré PW (2010) Choline and osmotic-stress tolerance induced in Arabidopsis by the soil microbe Bacillus subtilis (GB03). Mol Plant Microbe In 23:1097-1104 